\title{
An Historical Light Curve of CF Octantis from Digitised Images of the Bamberg Plate Archive
}

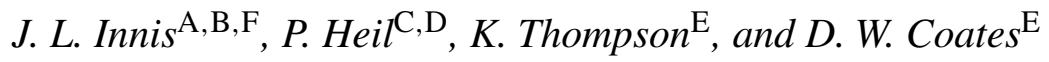 \\ A Geophysical Institute, University of Alaska Fairbanks, Fairbanks AK 99775, USA \\ B Present Address: Space and Atmospheric Sciences, Australian Antarctic Division, \\ Kingston TAS 7050, Australia \\ C IARC, University of Alaska Fairbanks, Fairbanks AK 99775, USA \\ D Present Address: TPAC, University of Tasmania, Hobart TAS 7001, Australia

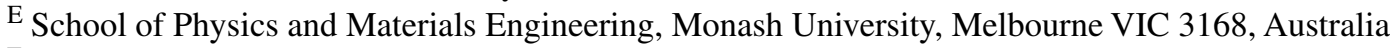 \\ F E-mail: john.innis@aad.gov.au
}

Received 2003 March 17, accepted 2004 February 16

\begin{abstract}
Digital images were made of Bamberg Observatory Sky Patrol plates of the field surrounding the active-chromosphere star CF Octantis (HD 196818). These images, taken with an inexpensive camera, were analysed using standard aperture photometry techniques. Good agreement was found with catalogued photographic magnitudes for stars in the range $m_{\mathrm{pg}} \sim 8.5$ to $\sim 10.5$. The root-mean-square deviations in the measured differences for non-variable field stars was found to be of order $0.10 \mathrm{mag}$, although a small number of larger differences occurred. For CF Oct, a period search of data from 1966 recovered the known $20 \mathrm{~d}$ variation due to starspot rotational modulation, with a range of variation of order 0.5 mag photographic. For active-chromosphere stars with moderately large photometric variations $(>\sim 0.3 \mathrm{mag}$ ), and moderate to long rotation periods ( $>\sim 1$ week), careful analysis of similarly obtained digital copies of archive plates may provide valuable insights into historical actvity.
\end{abstract}

Keywords: stars: activity — stars: individual (CF Oct) — stars: spots — techniques: photometric

\section{Introduction}

Astronomical plate archives provide an important data base for long-term studies of stellar variability. There is a growing interest in the use of such data bases, as demonstrated by, for example, Kroll et al. (1999); the Sofia Wide Field Plate Database (www.skyarchive.org); the Belgian Plate Archive Project (udapac.oma.be/ fido/ovid.html); and IAU resolution B3, 2000.

Intensive studies of active-chromosphere stars blossomed in the late 1970s and early 1980s following the recognition of the class, together with the combination of ground-based and spacecraft facilities suitable for this work. Candidate active stars were identified from spectroscopic surveys (e.g. Bidelmann \& MacConnell 1973; Hearnshaw 1979) or as X-ray sources from spacecraft missions. Detailed observational programs have given specific information on the properties of individual active stars, such as rotation rates, binarity, spectral line phenomena, and surface magnetic fields.

The photometric variability of a number of large amplitude active stars had been discovered by the Bamberg Southern Sky Patrol in the 1960s. The Bamberg plate archive is maintained by the $\mathrm{Dr}$ Remeis-Sternwarte (Remeis Observatory), Bamberg, Germany. This paper presents the results of a project which aimed to recover historical light curves of selected large amplitude active stars from digitised images of the plates. The images were obtained with an inexpensive digital camera, with only 8-bit resolution, but nevertheless indicate that for active stars of large photometric amplitude, and medium to long period, light curves can be found from plate archives.

The main target of our study was CF Octantis (HD 196818, $V \sim 8, \alpha \sim 20 \mathrm{~h} 50 \mathrm{~m}, \delta \sim-80 \mathrm{deg}$ ). It was discovered to be variable by the Bamberg Southern Sky Patrol, and was designated BV 893 (Strohmeier 1967). CF Oct is a K0 subgiant with a large and variable photometric modulation $(\sim 0.2-0.35 \mathrm{mag}$ in $V)$ with a $\sim 20.1 \mathrm{~d}$ period. Photometry of this star has been published by Innis et al. (1983), Lloyd Evans \& Koen (1987), Pollard et al. (1989), and Innis et al. (1997). Spectroscopic studies include those of Hearnshaw (1979), Collier (1982), and Innis et al. (1997). The star is probably single (Innis et al. 1997). It is a strong, flaring, microwave radio source (Slee et al. 1987a, b).

\section{Plate Data and Photometric Analysis}

\subsection{The Bamberg Observatory Plate Archive}

The Bamberg Southern Sky Patrol was undertaken by the Remeis Observatory from 1963 to 1976. Most plates were taken at Boyden, South Africa, but stations were also operated at St Miguel, Argentina and Mt John, New Zealand. A bank of cameras on a common mount obtained wide-field plates (approximately $13 \times 13$ degrees). The patrol plates 
were exposed for 1 hour, and recorded stars to magnitude 14. (For further information see Strohmeier \& Mauder 1969, and the on-line Wide Field Plate Database noted above.) The blue-sensitive emulsion (Agfa Gevaert 67 A50) produced standard photographic magnitudes $\left(m_{\mathrm{pg}}\right)$. Many variable stars were found and characterised (e.g. Schoeffel \& Koehler 1965). The plates have yielded a photometric precision near $0.05 \mathrm{mag}$, as demonstrated by iris photometer measurements by various workers (e.g. Schoffel 1964).

At our request, in late 2001 a check on the Bamberg card index by Horst Drechsel (Remeis Observatory) revealed that, in the 1960s, eight plates for CF Oct had been blinked and the variability noted, but no light curve had been produced. A subsequent visit by two of the authors (J.I. and P.H.) found CF Oct had been recorded on over 100 patrol plates. An analysis of a subset of these plates forms the current work. We believe the data presented here for CF Oct have not been previously published.

\subsection{The Plate Images}

Digital copies of the plates were obtained using an Intel 'pocket PC' camera connected to a laptop computer. VGA $(640 \times 480)$ images were collected as 24-bit (colour) TIFF files. The initial visit to Bamberg in 2002 January was limited to one day, and only plates from 1966 were copied. Plates were placed on a blink comparator and back-illuminated. Focussing the camera was achieved by inspecting the real-time image on the PC screen. At least four copies were made of each plate. The camera was held by hand above the plate. Small positional changes between exposures means each exposure represents an independent sampling of the plate. A sample image is shown in Figure 1. A small amount of distortion near the field edges arises from the camera optics. Analysis of these digital images (see below) indicated that satisfactory aperture photometry could be performed.

In a follow-up three day visit to the Remeis Observatory (2002 April/May) the camera was fixed in position above the blink comparator. The camera was located as close to the plate position as possible and yet still allow us to carefully insert and remove the fragile glass plates. However, physical constraints meant the camera was about three times as far from the plate as for the January (hand-held camera) images. (The images obtained in 2002 January were at an effective scale of $\sim 13 \operatorname{arcsec}_{\text {pixel }}{ }^{-1}$, while the later images were at $\sim 38 \operatorname{arcsec}$ pixel $^{-1}$.) A star image from the January data covered about nine times as many camera pixels as the later images (hence the sampling of the stellar profile was better suited to photometric measurements), and was obtained with a greater contrast, resulting in significantly better photometry. The scatter in the measurements from the April/May digital images is, unfortunately, too large to give useful photometry for CF Oct.

The TIFF images were converted to FITS images. As the photometric errors in the original plate material are of order 5\%, 8-bit precision appeared adequate. Inspection

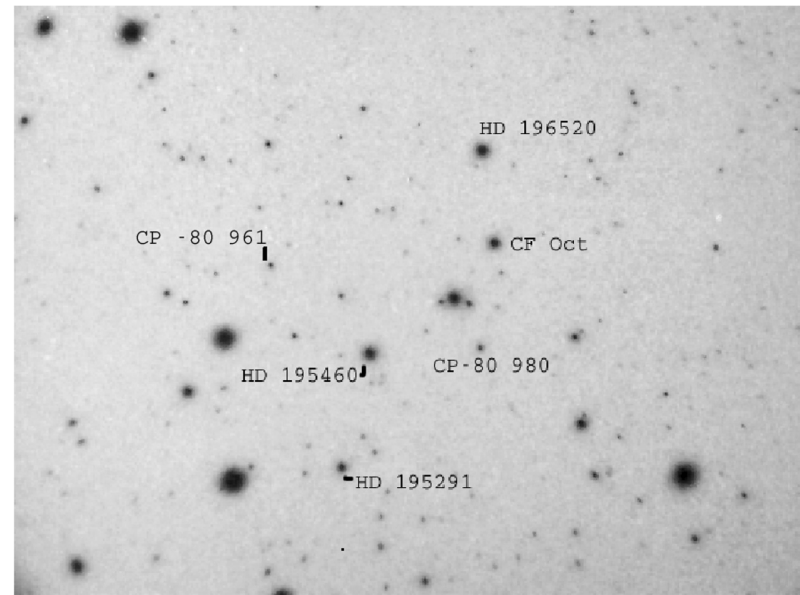

Figure 1 Sample image obtained with the Intel digital camera of part of Bamberg Sky Patrol plate Süd 18010. North is up, and east is to the left. The field shown is approximately $2.3 \times 1.7$ degrees. Stars of interest to the present work are identified. Reproduction here has resulted in a loss of quality compared to the original digital image.

of images of diffuse uniform illumination indicated that the camera field of view was uniform except very near the field edges, where some vignetting was noted (see above). No 'dark-count' correction has been applied. The images were converted to photo-positives using the IRAF task IMARITH to subtract each image from 255.

\subsection{Analysis with IRAF}

We chose to analyse digital images of the plates, rather than perform traditional iris photometry, as there are few operational iris photometers in existence - the Bamberg observatory photometer has not been used for many years. Burkholder (1995) obtained images of archival plates using a digital camera in a study of SN1974G, and used aperture photometry of the digital images to derive magnitude estimates. We largely followed this method in obtaining magnitude estimates from the Bamberg plate images, using the photometric tools in the IRAF task IMEXAMINE. It was necessary to choose comparison stars with care, and also to use appropriate values of the aperture radius and the sky annulus dimensions, to avoid contamination by nearby star images. We adopted an aperture radius of 8 pixels, and sky annulus inner and outer radii of 11 and 16 pixels respectively, for the analysis. Note that the star aperture, of radius 8 pixels, contains around 200 pixels, while the sky annulus (11-16 pixel radius) contains just over 400 pixels. The adopted sky value was the median of the pixel values in the annulus.

\subsection{Camera Uniformity and Emulsion Nonlinearity}

There are several issues of potential concern in using digitised images such as these to obtain photometric measurements. Any non-uniformity in the camera response over the field of view, such as vignetting in the camera optics or large pixel-to-pixel variations on the camera CCD, may affect the measure. The non-linear response 
of the photographic plate (and potentially of the digital camera as well) must also be accounted for.

The former concerns, non-uniformity over the field of view and from pixel to pixel, are usually dealt with using flat-field exposures. No flat field exposures were obtained in the initial visit to Bamberg, but during the later April/May trip a number of exposures were obtained of the illuminated blink comparator screen, which provided an approximation to a diffuse, uniform field. Note that the camera-operating software automatically set the exposure levels. Analysis of the images showed that the pixel-topixel variation was very small, and corresponded to the digitisation noise ( \pm 1 bit). That is, for a 'pixel count' of 220 bits, the noise was \pm 1 bit, or $0.5 \%$. There was slight vignetting over the $640 \times 480$ pixel field of view. However the vignetting was less than $10 \%$ over a near-central $550 \times 400$ area, and was always less than $20 \%$ except at the very corners of the field. CF Oct and the primary comparison star of HD 196520 were well within the area of low vignetting. As the vignetting loss is comparable to the expected plate precision, and as these 'flat-fields' were not obtained simultaneously with the images we have analysed, we have not applied any correction to the images.

It is also worth noting that, as the Bamberg plates are one-hour exposures, image motion, guiding errors, optical aberrations, focus irregularities, and so forth all contribute to give a stellar image much larger than the instantaneous seeing disk. The typical radii of the stellar images at halfmaximum for CF Oct were of order 3-4 digital camera pixels (i.e. a FWHM of 6-8 pixels). That is, the star image returns significant 'counts' ( $\geq 50 \%$ of the peak) in some 30 to 40 pixels. Such a large number of pixels in the aperture photometry also reduces the requirement to flat field, as small random pixel-to-pixel variations are largely eliminated in the sum.

Linearity is a significant issue for photographic photometry. The darkening of photographic emulsion by a stellar image is known to be inherently nonlinear process. However, linear transformations can be obtained between measurements from digitised plate images and catalogued magnitudes, as shown by Burkholder (1995). To see if similar satisfactory transformations could be derived for the Bamberg plates over the smaller magnitude range of interest for this work, we measured ten field stars ranging from $m_{\mathrm{pg}} 8.5$ to 10.5 on two plate images (Süd 10738 and Süd 10810). We adopted the faintest star (CP -80980 , $\left.m_{\mathrm{pg}} \sim 10.44\right)$ as the reference, deriving photographic magnitudes of the other nine stars differentially to the catalogued value for CP -80980 . For one star, CP -81890 , only one measurement could be obtained, as the star was at the frame edge in the other case.

Plotting the derived aperture photometry magnitudes against catalogued magnitude (Figure 2) shows the data are well correlated. The error bar represents the full range in the measurements of each star. The line in this figure has a $1: 1$ gradient, and shows the relationship expected for equality of the two quantities. It is not a fit to the data. However, the data points appear well described by this

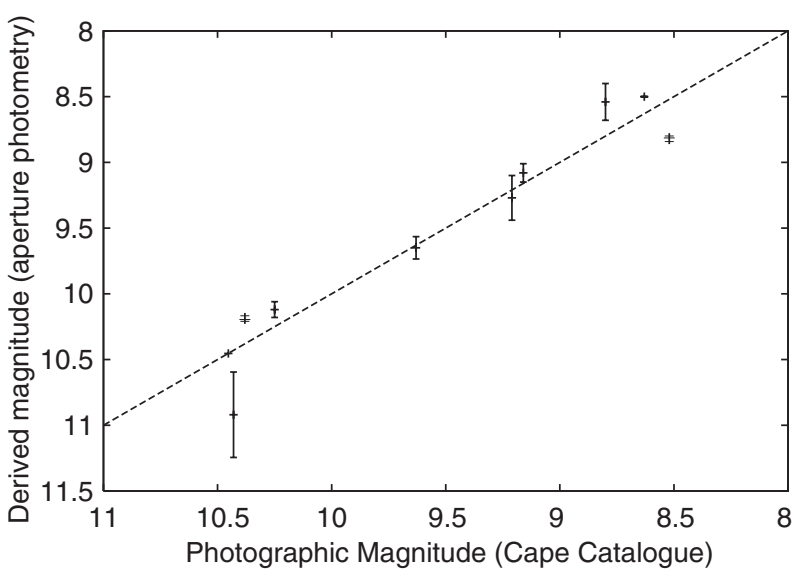

Figure 2 Linear plot showing derived photographic magnitudes compared to those listed in the Cape Photographic Catalogue (Stoy 1966, 1968), averaged from two plate images. The derived magnitudes have been determined relative to the mean of the observations of CP -80980 . The line is of $1: 1$ gradient, and is not a fit to the data.

line, given the experimental errors, which suggests there is no significant nonlinearity in the derived photographic magnitudes for the range shown.

We carried out this multistar analysis for two plates only, and hence have not in principle checked the linearity for all plates we used. However, we did measure a number of stars on each image of each plate. Results for selected stars (including CP -80980) given as differential magnitudes with respect to HD 196520 (= CP -80978), are presented in the next Section. The internal consistency of these results suggested to us that the two plates analysed in detail were typical of the remainder. We conclude that, to the precision of the plates, and over this approximately two-magnitude range, the magnitudes found from the aperture photometry could be used directly, being only offset by a constant amount from the catalogued photographic magnitudes.

CP $-80961\left(m_{\mathrm{pg}}=10.43\right)$ appears discrepant, and the range in the two measurements for this star is much greater than for any other. This may be related to the camera we used, but we have not identified the cause. Except for the few such exceptional cases noted below, the simple aperture photometry appears to give reliable photographic magnitudes for stars in the range $m_{\mathrm{pg}}$ from $\sim 8.5$ to 10.5 . (The total range, including observational scatter, we found for the target star CF Oct was about 8.5 to $9.3 \mathrm{~m}_{\mathrm{pg}}$.)

The standard deviation in the differences (aperture photometry minus catalogued value) is $0.25 \mathrm{mag}$. Omitting the datum for CP -80961 gives a standard deviation of $0.17 \mathrm{mag}$. If random errors dominate, we may expect a statistical error near $0.1 \mathrm{mag}$ when averaging four measurements. As shown in the following Section, an experimental precision near this value appears reasonable.

\section{Results of the Photometry}

\subsection{CF Oct in 1966}

We determined magnitudes of CF Oct and three field stars (HD 195291, HD 195460, and CP -80980) relative 

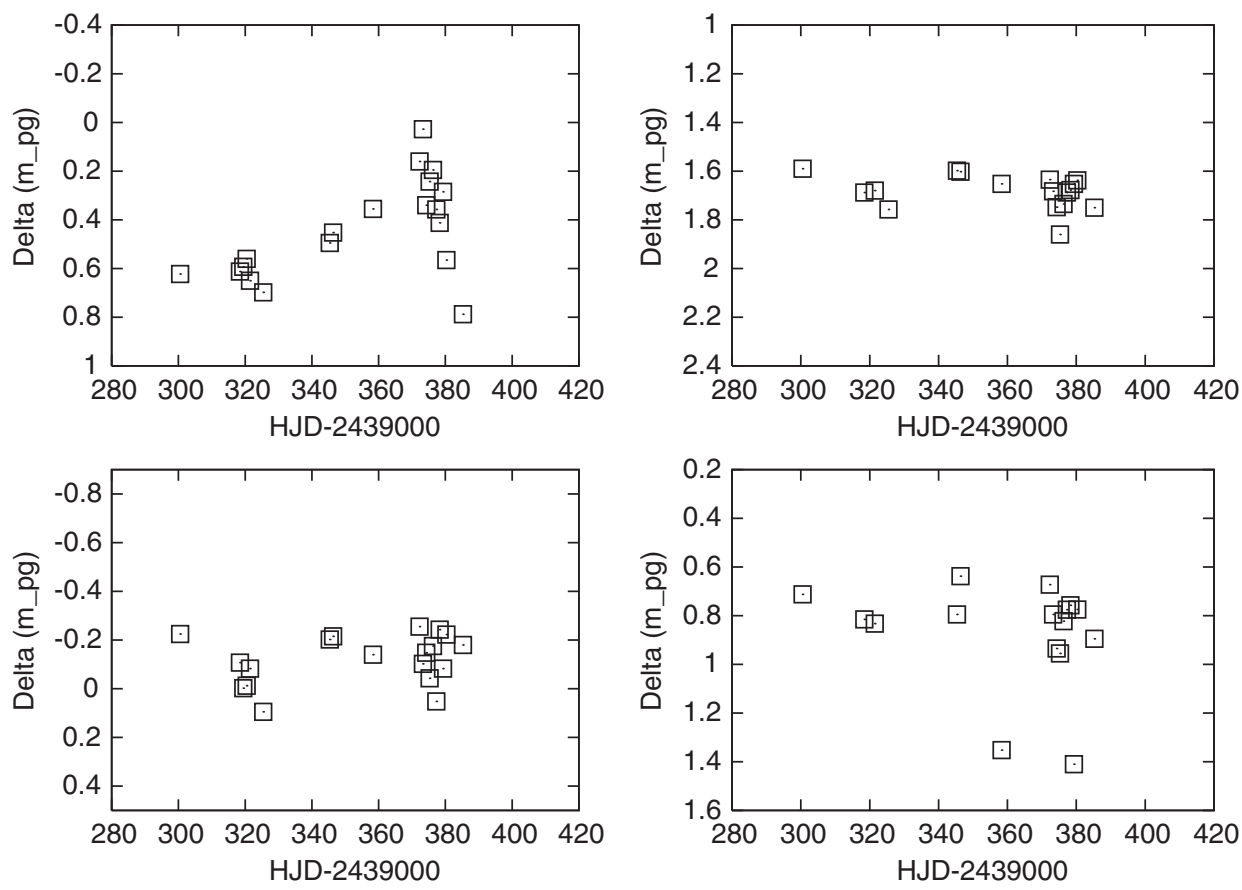

Figure 3 Results of the aperture photometry, shown as differential photographic magnitudes relative to HD 196520. Clockwise from top left: CF Oct; CP -80980; HD 195291; HD 195460.

Table 1. Mean magnitude differences and standard deviations with respect to HD 196520

\begin{tabular}{lrc}
\hline Star & Mean & Standard deviation \\
\hline CF Oct & 0.44 & 0.20 \\
CP - 80980 & 1.68 & 0.07 \\
HD 195460 & -0.12 & 0.10 \\
HD 195291 & 0.87 & 0.22 \\
(HD 195291 & 0.80 & 0.09 omitting two points)
\end{tabular}

to HD 196520 ( $\left.=\mathrm{CP}-80978, m_{\mathrm{pg}}=\sim 8.52\right)$, a K-type star, at small angular separation from CF Oct, used as the comparison star in the photometric studies noted in Section 1. Each of the four images of a given plate was analysed separately, and the results averaged. This averaging reduces the noise introduced when digitally sampling the plates.

The results of the aperture photometry are summarised in Figure 3. The three non-variable stars show little change greater than observational scatter, while for CF Oct a slow rise and faster fall is evident. Measurements of the star HD 195291 on three plates and measurements of the star CP -80980 on two plates were wildly discrepant, and have been omitted from further consideration as they fall far outside the range of the plots. Two other measurements of HD 195291 are around 0.6 mag fainter than the remaining data (these data are shown). Inspection of the individual frames did not reveal any clear reason for this.

The mean and standard deviations of the magnitude differences of CF Oct and the three field stars, relative to HD 196520, are shown in Table 1. The reduced scatter in the differential magnitudes for CP -80980 compared with
Table 2. Magnitude differences of CF Oct with respect to HD 196520

\begin{tabular}{lcc}
\hline $\begin{array}{l}\text { Plate no. } \\
\text { Süd }\end{array}$ & HJD -2439000.0 & $\Delta m_{\mathrm{pg}}$ \\
\hline 10371 & & \\
10486 & 300.501 & 0.62 \\
10522 & 318.448 & 0.61 \\
10558 & 319.455 & 0.59 \\
10594 & 320.455 & 0.56 \\
10636 & 321.464 & 0.65 \\
10738 & 325.431 & 0.70 \\
10762 & 345.352 & 0.50 \\
10810 & 346.355 & 0.45 \\
10888 & 358.324 & 0.36 \\
10900 & 372.281 & 0.16 \\
10918 & 373.282 & 0.03 \\
10942 & 374.285 & 0.34 \\
10972 & 375.285 & 0.24 \\
11002 & 376.285 & 0.20 \\
11020 & 377.285 & 0.36 \\
11068 & 378.284 & 0.42 \\
11092 & 379.291 & 0.29 \\
11182 & 380.285 & 0.57 \\
& 385.244 & 0.79 \\
\hline & &
\end{tabular}

those for HD 195460 and HD 195291 may be related to the angular distance between the given star and HD 196520 (refer to Figure 1). Hence some form of flat-field treatment of the background illumination may be of benefit in future analysis. Omitting the two discrepant points shown for HD 195291 in Figure 3 reduces the standard deviation significantly. From the results in Table 1 we believe that, under favourable circumstances, magnitude differences can be obtained to better than 0.1 magnitude standard deviation. 


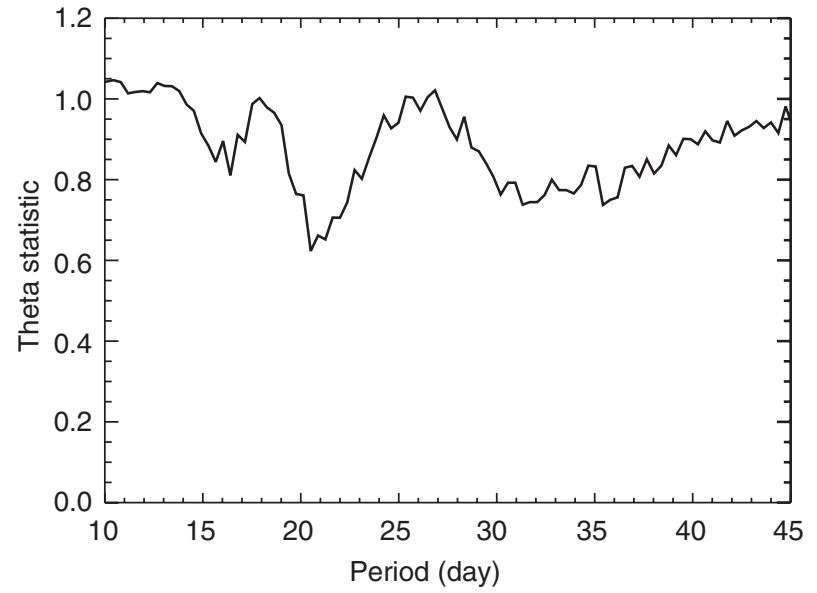

Figure 4 PDM spectrum of the CF Oct photometry from the 1966 Bamberg Patrol plates. A minimum in the $\theta$ statistic near $20 \mathrm{~d}$ coincides with the known $20.15 \mathrm{~d}$ photometric variation of this star.

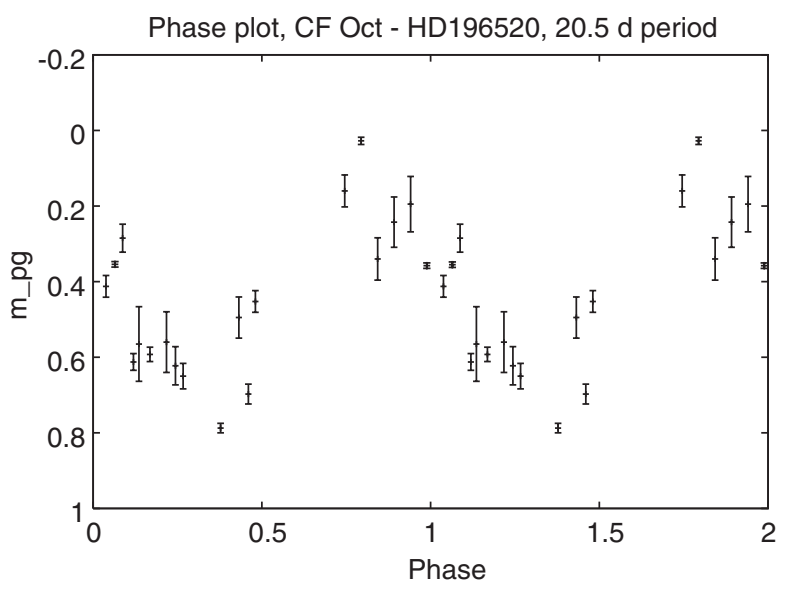

Figure 5 Phase plot for CF Oct, for 1966, as differential photographic magnitudes with respect to HD 196520, from the Bamberg plate images, using a period of $20.5 \mathrm{~d}$ found from the PDM spectrum, and the epoch of Innis et al. (1997). Data are repeated to show the variation more clearly. Error bars represent the standard deviation in the photometry from the four digital images made of each plate.

The final photographic photometry of CF Oct for 1966 , determined by the above method, is summarised in Table 2. The Süd (south) plate number and the HJD of mid-exposure (from listings on the plate envelopes) are shown, along with the differential photographic magnitude expressed relative to HD 196520.

\subsection{Light Curve Analysis}

A spectral analysis of the magnitude differences between CF Oct and HD 196520 was performed using the phase dispersion minimisation (PDM) method of Stellingwerf (1979). The resulting PDM spectrum is shown in Figure 4. A minimum in phase dispersion is detected for a period $\sim 20 \mathrm{~d}$. The value of the period found from the PDM analysis varies slightly depending the input search parameters, but is in the range 19 to $21 \mathrm{~d}$ for reasonable search values. A period of $20.5 \mathrm{~d}$ is found from a fine scan using four 'covers' (Stellingwerf 1979) when searching the range from 10 to $45 \mathrm{~d}$. We estimate the error to be near $\pm 0.5 \mathrm{~d}$. As noted earlier, photoelectric photometry by a number of workers has indicated CF Oct varies with a period near $20.15 \mathrm{~d}$. The exact rotational periods of stars such as CF Oct are very hard to determine. The photometric variation is believed to be due to starspot activity. Changes in spot configuration, spot size, and probably spot temperature all contribute to changes in the light curve, making it difficult, if not impossible at times, to combine data from different epochs to refine the period. CF Oct is known to exhibit significant light-curve variations. Additionally there is the possibility of differential rotation on the stellar surface, meaning that no single period could ever be determined if the star spots appear at different latitudes at different times. We are very confident that the PDM analysis of the aperture photometry presented here has detected a real variation of CF Oct. For the reasons noted above, we believe the slight difference between the formal value of the period derived in this work and that in Innis et al. (1997) is of no consequence.

Figure 5 shows the light curve of CF Oct for 1966 using a period of $20.5 \mathrm{~d}$, and epoch of HJD 2445199.5 (as used by Innis et al. 1997). The error bars represent the standard deviation found from the measurements of the separate images of each plate. CF Oct varies by about $0.5 \mathrm{mag}$. The star has often shown modulation of 0.2 mag or more in $V$ light (see references noted in Section 1). In particular, a large amplitude modulation was exhibited in 1983-1984, when the range was $\sim 0.4 \mathrm{mag}$ in $V$, as seen in photoelectric photometry presented by Innis et al. (1997). Multicolour photometry obtained at that time showed that the amplitude in $B$ was slightly larger, at around $0.45 \mathrm{mag}$. The photographic magnitudes obtained for this present study would be closer to $B$ than $V$ magnitudes, hence a variation near $0.5 \mathrm{mag}$ is not unprecedented.

The three light curves in Innis et al. (1997) showed a relatively stable phase of minimum light near phase 0.5 for the years 1982-1986. Plotting the 1966 photographic photometry with the same period (20.15d) and epoch as in Innis et al. (1997) gives minimum light near phase 0.45 (plot not shown). Whether this is coincidence or indicates a long-lived active longitude on CF Oct we hope to be able to resolve from further study of the archival data.

\section{Discussion}

We believe we have demonstrated that it is possible to perform reliable aperture photometry of digital images of the Bamberg plates using a relatively unsophisticated camera and analysis process. The derived photographic magnitudes appear linear over the measured range $m_{\mathrm{pg}} \sim 8.5$ to $\sim 10.5$. This should extend to fainter stars as well, given that analogue measurements have been shown to be valid to fainter limits (e.g. Schoeffel \& Koehler 1965).

Using a camera to digitise small areas of interest from archival plates offers some advantages over the use of a scanner. The camera is cheaper (a few hundred dollars compared to thousands of dollars for a scanner), and 
quick - once the camera is set up in position over the field of interest images can be obtained as fast as the operator can position the plates. There are clear improvements that could be incorporated in our method, such as the use of a scientific grade camera, ensuring optimal sampling of the stellar images on the plates, obtaining flat-fields (of the illuminating screen), and with images being saved directly as 16-bit FITS files to preserve precision and to avoid the inconvenience of file conversion.

With attention to detail, and with close attention to matters such as choice of aperture radius and comparison stars, it should be possible to use this method to study stars of moderately large photometric amplitudes, given a noise level near $0.1 \mathrm{mag}$. The patrol plates generally covered a given field only once on any particular night, hence active stars with highly variable light curves (from spot changes) and/or periods less than about a week or so may not be well sampled.

\section{Conclusion}

Digital images of a section of the Bamberg Southern Sky Patrol plates were obtained using a cheap, commercially available digital camera. Simple aperture photometry of the resulting images for stars in the field of CF Oct indicated good agreement between the derived and catalogued photographic magnitudes for stars in the range $m_{\mathrm{pg}} \sim 8.5$ to 10.5 . The rms differences between several non-varying field stars was found to be around $0.1 \mathrm{mag}$. CF Oct showed systematic photometric changes. A spectral analysis recovered the known $20 \mathrm{~d}$ rotational modulation of this star, and the data indicated that a large-amplitude variation, near $0.5 \mathrm{mag}\left(m_{\mathrm{pg}}\right)$, was present in 1966 .

We believe that, with care, use of such a technique to digitise small areas of wide-field plates is a viable means of retrieving archival photometric histories of certain objects. The use of a more suitable camera, allowing greater control of the exposure, and retaining greater than 8-bit resolution in the images, would be advantageous.

\section{Acknowledgments}

J.I. and P.H. thank the Remeis Observatory, Bamberg, for access to the plate collection and for hospitality. We especially thank Irmela Bues, Horst Drechsel, and Rainer Sterzer (Remeis Observatory). We also acknowledge the staff of the Remeis Observatory who conceived and performed the Southern Sky survey, and carefully archived the plates. We thank the referees for their comments. This work has made use of the NASA ADS bibliographic database, the SIMBAD database at the CDS Strasbourg, and the Wide Field Plate Database (www.skyarchive.org). The reduction software IRAF, from the US National Optical Astronomy Observatories, was used in the analysis.

\section{References}

Bidelmann, W. P., \& MacConnell, D. J. 1973, AJ, 78, 687

Burkholder, V. 1995, JAAVSO, 23, 127

Collier, A. C. 1982, PhD Thesis (Christchurch: Univ. of Canterbury) Hearnshaw, J. B. 1979, Changing Trends in Variable Star Research, Proc IAU Colloq. 46, eds. F. M. Bateson, J. Smak, \& I. H. Urch (Hamilton: Univ. of Waikato), 371

Innis, J. L., Coates, D. W., Dieters, S. W. B., Moon, T. T., \& Thompson, K. 1983, IBVS, 2386

Innis, J. L., Coates, D. W., \& Thompson, K. 1997, MNRAS, 285, 515

Kroll, P., la Dous, C., \& Brauer, H.-J. (eds.) 1999, Treasure-Hunting in Astronomical Plate Archives (Frankfurt am Main: Deutsch)

Lloyd Evans, T., \& Koen, M. C. J. 1987, SAAO Circ., 11, 21

Pollard, K. R., Hearnshaw, J. B., Gilmore, A. C., \& Kilmartin, P. M. 1989, JA\&A, 10, 139

Schoffel, E. 1964, IBVS, 71

Schoeffel, E., \& Koehler, U. 1965, IBVS, 100

Slee, O. B., Nelson, G. J., Stewart, R. T., Wright, A. E., Innis, J. L., Ryan, S. G., \& Vaughan, A. E. 1987a, MNRAS, 229, 659

Slee, O. B., Nelson, G. J., Stewart, R. T., Wright, A. E., Jauncey, D. L., Vaughan, A. E., Large, M. I., Bunton, J. D., Peters, W. L., \& Ryan, S. G. 1987b, PASA, 7, 55

Stellingwerf, R. F. 1979, ApJ, 227, 935

Stoy, R. H. 1966, Ann. Cape Obs., 21

Stoy, R. H. 1968, Ann. Cape Obs., 22

Strohmeier, W. 1967, IBVS, 178

Strohmeier, W., \& Mauder, H. 1969, Sky. Tel., 37, 10 